Revista de Derecho Público: Teoría y Método Marcial Pons Ediciones Jurídicas y Sociales

Vol. 2 | 2020 pp. 51-72
Madrid, 2020

DOI: 10.37417/RPD/vol 22020340

(C) Joseba Fernández Gaztea ISSN: $2695-7191$

Recibido: $25 / 05 / 2020$ | Aceptado: 10/08/2020

Editado bajo licencia Creative Commons Attribution 4.0 International License.

\title{
CONCEPTO Y FUNCIONES DE LA TÓPICA JURÍDICA EN EL DERECHO PÚBLICO*
}

\section{CONCEPT AND FUNCTIONS OF LEGAL TOPICS IN PUBLIC LAW}

\author{
Joseba Fernández Gaztea \\ Profesor de Derecho Administrativo \\ Universidad de Navarra
}

RESUMEN: En torno a la tópica jurídica se siguen planteando los interrogantes de qué es, cuál es la naturaleza de su objeto material -los tópicos-, y en qué puede ser de utilidad al Derecho. La primera parte de este artículo tiene por objeto el concepto de tópica jurídica y de tópicos jurídicos. La segunda se apoya en las conclusiones de la primera para sugerir cuatro funciones de la tópica en el Derecho público.

PALABRAS CLAVE: tópica; tópica jurídica; tópicos jurídicos, Derecho público.

ABSTRACT: The questions of what is legal Topics, what is the nature of its material object (topics), and in which way can it be useful to the law, continue to be raised. The object of the first section of this article is the concept of legal Topics and its object, legal topics. The second one takes advantage of the conclusions of the first to suggest four functions of Topics in public law.

KEYWORDS: topics; legal Topics; public law.

* Este artículo es fruto de la comunicación presentada en el Seminario de Teoría y Método STEM que tuvo lugar en la Universidad de Navarra el 29 de noviembre de 2019. La investigación previa se llevó a cabo en la biblioteca de la Westfälische Wilhelms-Universität de Münster con el apoyo financiero de una beca generosamente concedida por la fundación Karina und Erich Schumann Stiftung.

En el texto se abrevia la palabra editor con ed.; director con dir.; ibídem con ibid.; página con p.; opus citatum con op. cit., $y$; volumen con vol. 
SUMARIO: 1. INTRODUCCIÓN.-2. EL CONCEPTO DE TÓPICA JURÍDICA: 2.1. La tópica. 2.2. La tópica jurídica. 2.3. La indeterminación de la tópica: 2.3.1. La indeterminación de la tópica: los tópicos. 2.3.2. La indeterminación de la tópica: la tópica como intento por explicar el proceso de razonamiento en el ámbito de lo plausible y discutible.-3. LAS FUNCIONES DE LA TÓPICA JURÍDICA: 3.1. Auxilio para entender la racionalidad de la norma positiva. 3.2. Nexo entre los distintos elementos de una unidad jurídica plural. 3.3 Apoyo para la innovación y argumentación jurídicas. 3.4. Función pedagógica.-4. CONCLUSIONES.— 5. BIBLIOGRAFÍA

\section{INTRODUCCIÓN}

Después de Topik und Jurisprudenz ${ }^{1}$ de Viehweg la tópica jurídica es un tópico metodológico ineludible. Veinte años desde que la retórica y la tópica se recuperaran para las ciencias sociales, solo el número de escritos en lengua alemana dedicado a ambas rondaba los $2 \cdot 200^{2}$. Como indica un artículo reciente de esta publicación, la tópica sigue siendo de interés. ${ }^{3}$.

De entre todas las preguntas en torno a la tópica, las más fundamentales son susceptibles de más reflexión porque no han recibido respuesta concluyente; así se desprende de la literatura. Estas preguntas son qué es la tópica y para qué sirve: el concepto y las funciones de la tópica ${ }^{4}$. Con este artículo se quiere analizarlas desde una perspectiva jurídico-pública: tratar el concepto y funciones de la tópica jurídica en el Derecho público.

\section{EL CONCEPTO DE TÓPICA JURÍDICA}

\subsection{La tópica}

La tópica es, esencialmente, una técnica de búsqueda de premisas para la argumentación. Comprende también el estudio de la naturaleza de esas premisas y el uso que se hace de ellas 5 . Se desarrolla por medio de la discusión y no de la deducción,

1 Theodor VIEHWEG, Topik und Jurisprudenz, C. H. Beck, München, 1953.

2 Robert JAMISON y Joachim DYCK, Topik, Argumentation, Frommann-Holzboog, Stuttgart/ Bad Cannstatt, 1983. Este libro consiste en un largo catálogo de escritos que en sí mismo es testimonio de la gran cantidad de bibliografía académica que entre los años 1960 y 1980 se ocupó de la materia. Adviértase que este catálogo incorpora referencias de obras que se ocupan de la tópica más allá del Derecho; por ejemplo de la tópica en la literatura o en la ciencia política. El número exacto de referencias listadas es de 2.185 .

3 Silvia DÍEZ SASTRE, "La tópica como método en el Derecho público", Revista de Derecho Público: Teoría y Método, vol. 1, 2020, pp. 363-396.

4 "There are just two serious questions about loci, (1) what is a locus, and (2) what is it good for", en Sten EBBESEN, "The Theory of loci in Antiquity and the Middle Ages", en Klaus JACOBI (ed.), Argumentationstheorie - Scholastische Forschungen zun den logischen und semantischen Regeln korrekten Folgerns, E. J. Brill, Leiden/New York/Köln, 1993, pp. 15-40 (p. 29).

5 Este esquema de triple significado de la tópica como, (i) técnica de búsqueda de premisas, (ii) teoría sobre la naturaleza de estas premisas, y (iii) teoría sobre el uso de estas premisas, está tomado de 
razón por la cual se entiende por tópica, también, una investigación que discurre por el cauce de la dialéctica y el consenso, y no de la deducción sistemática a partir de primeras verdades ${ }^{6}$.

Es en el campo de las ciencias de la razón práctica donde la tópica es de utilidad ${ }^{7}$. Es decir, la tópica está al servicio del conocimiento del obrar humano para orientar su conducta. Su objeto son proposiciones plausibles -no demostrables- y, en consecuencia, discutibles. Las premisas objeto de la tópica no son, por tanto, apodícticas ${ }^{8}$ : no sirven para elaborar un razonamiento analítico basado en proposiciones verdaderas de por sí -como sí sirven las de la lógica formal-, sino para elaborar un razonamiento dialéctico ${ }^{9}$ por medio de entimemas (silogismos en los cuales o bien no se enuncian todas las premisas, o bien se parte de premisas verosímiles o plausibles). Como el resto del arte e investigaciones aristotélicos, en su origen la tópica se dirige a la consecución de un bien ${ }^{10}$.

De entre aquellas premisas que son objeto de la tópica, algunas se tienen por generalmente aceptadas, y, precisamente, por esa generalidad de su aceptación se las figura como lugares compartidos o comunes a los interlocutores: loci o topoi. Son los tópicos. Se ha afirmado que este término abarca "cualquier dato comunicativo que comparten los intercomunicantes en un momento dado, en un colegio cualquiera" ${ }^{11}$. Pueden ser un tópico, entre otros, un concepto, una tesis normalmente aceptada, un principio, una descripción, un argumento, o una regla.

Todo indica que el origen de la tópica se encuentra en la dialéctica y retórica aristotélicas ${ }^{12}$; las evidencias de investigaciones anteriores al estagirita son más bien frágiles ${ }^{13}$. Sería Cicerón quien trataría de poner al servicio del jurista las ventajas de una

Manuel ATIENZA, Las razones del Derecho - Teorías de la argumentación jurídica, Centro de Estudios Constitucionales, Madrid, 1997, p. 57.

6 Manuel Jesús RÓDRIGUEZ PUERTO, “Tópica y humanismo jurídico", Anuario de Filosofía del Derecho, 2001, pp. 363-383 (p. 364).

7 ARISTÓTELES, Tratados de lógica (Órganon) I, Gredos, Madrid, 1982 (traducción de Miguel Candel Sanmartín y José Montoya), pp. 89-92.

8 Cecilia Ana LERTORA MENDOZA, "La tópica en la lógica jurídica”, Anuario de Filosofía del Derecho, vol. 18, 1975, pp. 203-222 (p. 207).

9 ARISTÓTELES, 1982: 90.

${ }^{10}$ La idea de que todo arte e investigación se dirige a un bien es aristotélica. Véase ARISTÓTELES, Ética Nicomaquea - Ética Eudemia, Libro I, Gredos, Madrid, 1993 (traducción de Julio Pallí Bonet y José Ignacio García Armendáriz).

${ }_{11}$ Francisco PUY MUÑOZ, "Tópica jurídica y retórica jurídica: un ensayo de distinción”, Ars Iuris, vol. 31, 2004, pp. 271-303 (p. 281). Véase también la definición de este autor en Francisco PUY MUÑOZ, Tópica jurídica, Paredes, Santiago de Compostela, 1984, pp. 807-808.

12 Particularmente importantes son tres de sus obras: Tópicos (una de las obras recopiladas en el denominado Órganon), Retórica, y también parte de su Metafísica. Véase el comentario a este respecto en Niels Jørgen GREEN-PEDERSEN, The Tradition of the Topics in the Middle Ages - The Commentaries on Aristotle's and Boethius' "Topics" Philosophia Verlag, München/Wien, 1984, p. 13.

13 Ferdinand SCHUPP, "Zur Geschichte der Beweistopik in der älteren griechischen Gerichtsrede", Wiener Studien, vol. 45, 1926, pp. 17-28, y EBBESEN, 1993: 13. 
tópica aristotélica reelaborada ${ }^{14}$, y después Boecio quien por medio de In Ciceronis Topica y De differentiis topicis haría de nexo principal entre la tópica de Cicerón y la tópica medieval, dominando así la obra de Boecio la teoría de la argumentación ${ }^{15}$ en la Europa medieval desde aproximadamente el año 1050 hasta el siglo xv, momento en el cual la tópica aristotélica ya estaba extendida por las universidades ${ }^{16}$.

Durante la Baja Edad Media se seguía empleando la tópica como herramienta de búsqueda de conocimiento ${ }^{17}$, para quedar después relegada a un segundo plano con la progresiva asimilación de los postulados cartesianos. En este nuevo escenario, autores como Vico sostuvieron la conveniencia del empleo de la tópica ${ }^{18}$. Después de la Segunda Guerra Mundial, se empezó a hacer referencia a una resurrección de la tópica en materias como la ciencia política, la sociología, la teoría literaria, la filosofía o la jurisprudencia ${ }^{19}$. En la década de los setenta del siglo xx la incapacidad de llegar a un acuerdo sobre su contenido la relegó a un segundo plano ${ }^{20}$.

${ }^{14}$ Manfred FUHRMANN, "Die zivelrechtlichen Beispiele in Ciceros Topik", en Thomas SCHIRREN y Gert UEDING (eds.), Topik und Rhetorik, Max Niemeyer, Tübingen, 2000, pp. 51-66. Una explicación de los conceptos fundamentales de la Topica de Cicerón en Alejandro GUZMÁN BRITO, "Dialéctica y retórica en los 'topica' de Cicerón", en Alfonso CASTRO SÁENZ y Fernando LLANO ALONSO (eds.), Cicerón - El Hombre y los Siglos, Comares, Granada, 2016, pp. 267-304. Se conoce más bien poco de la literatura escrita en el período que transcurrió entre Aristóteles y Cicerón, de la cual destacan las citas de la obra de Teofrasto contenidas en el comentario de los Tópicos de Aristóteles de Alejandro de Afrodisias. Véase GREEN-PEDERSEN, 1984: 37.

15 Heine HANSEN, "Boethius' De topicis differentiis, Commentaries on", en Henrik LAGERLUND (ed.), Encyclopedia of Medieval Philosophy - Volume 1-A-L, Springer, New York, 2011, pp. 176177 (p. 176).

${ }_{16}$ Un estudio detallado de los autores y comentarios que dominaron la tópica medieval hasta el empleo de Aristóteles en las universidades europeas se encuentra en GREEN-PEDERSEN, 1984. Se han de tener presentes conclusiones más recientes que indican que la introducción de los tópicos ciceronianos en la Europa medieval no fue obra exclusiva de Boecio. Fueron también relevantes, por ejemplo, los comentarios de Marciano y Casiodoro al comentario de los tópicos de Cicerón de un autor cuya obra no nos ha llegado: Mario Victorino; también lo fueron los comentarios a los tópicos de Cicerón de Isidoro de Sevilla, o la obra de Alcuino de York. Una explicación detallada, en Fiorella MAGNANO, "Cicero's Lists of Topics from Antiquity to the Early Middle Ages", Revista Española de Filosofia Medieval, vol. 22, 2015, pp. 85-118.

${ }_{17}$ En relación con la tópica de esta etapa histórica, véase RODRÍGUEZ PUERTO, 2001; Günter FRANK, "Traditionsfähigkeit antiken Wissens in der Frühen Neuzeit - Zur Überlieferung der aristotelishcen Ethica", en Thomas FRANK, Ursula KOCHER y Ulrike TARNOW (eds.), Topik und Tradition-Prozesse der Neuordnung von Wissensüberlieferungen des 13. bis 17. Jahrbunderts, V\&R unipress, Göttingen, 2007, pp. 175-190, y SCHIRREN y UEDING, 2000: 91-272.

18 Theodor VIEHWEG, Tópica y jurisprudencia, Taurus, Madrid, 1964 (traducción de L. Díez Picazo Ponce de León), pp. 25-29; Juan VALLET DE GOYTISOLO, "La jurisprudencia y su relación con la tópica en la concepción de Giambattista Vico”, Revista de Estudios Políticos, vol. 206-207, 1976, pp. 77-136, y Ramón BELTRÁN CALFURRAPA, "La tópica jurídica y su vinculación argumentativa con el precedente y la jurisprudencia", Revista de Derecho de la Pontificia Universidad Católica de Valparaiso, vol. 39, 2012, pp. 587-606 (pp. 593-594).

19 ATIENZA, 1997: 50.

20 DÍEZ SASTRE, 2020: 366, número marginal 5. 
En esta última versión de la tópica se quiso ver una expresión actualizada del modo tradicional de contrastar la verdad en Occidente. Desde esta perspectiva, la tópica contemporánea se habría originado en la Antigüedad clásica y persistido en la Europa medieval hasta bien entrado el siglo XviII, período en el cual se le puso entre paréntesis hasta pasada la Segunda Guerra Mundial por influencia del racionalismo cartesiano, la filosofía crítica de Kant y el positivismo ${ }^{21}$.

No obstante la continuidad de este relato, es necesario advertir que no se pueden obviar las cesuras acaecidas a lo largo de los siglos precedentes sin arriesgar caer en cierta imprecisión ${ }^{22}$. En particular, hay que apuntar que la tópica del siglo xx se desenvolvió en el seno de una red conceptual distinta a la de la tópica aristotélica o medieval ${ }^{23}$. Se debe tener presente, por ejemplo, que la tópica de Viehweg surgió sobre el fundamento de una doctrina -la filosofía de Heidegger y su círculo hermenéutico ${ }^{24}$ - que no había formado parte de contextos conceptuales anteriores como fueron el clásico y medieval.

\subsection{La tópica jurídica}

En el contexto particular de la Alemania de la posguerra, Viehweg procedió como se había hecho en los campos de la política, la sociología, la teoría literaria y la filosofía, y trasladó al Derecho el nuevo planteamiento tópico de Curtius ${ }^{25}$. Propuso rescatar la tópica clásica para la jurisprudencia partiendo de la constatación de que el Derecho comienza a partir de problemas concretos -aporías-, y no de un sistema ${ }^{26}$. Estos problemas requieren una solución para el caso, y ésta se encuentra a través de un proceso dialógico que necesita del auxilio de los tópicos. En tanto que en cada supuesto las circunstancias marcan un punto de inicio único y distinto, la utilidad para el Derecho del sistema y del proceder deductivo parece desvanecerse.

${ }^{21}$ Franz WIEACKER, “Theorie des Rechtes und der Rechtsgewinnung”, en Dieter SIMON (ed.), Franz Wieacker-Ausgewählte Schriften - Band 2, Metzner, 1983, pp. 81-100 (p. 82).

22 Una aproximación a la historia conceptual en Reinhart KOSELLECK, Futuro pasado, Barcelona, 1993, y; Elías José PALTI, "From Ideas to Concepts to Metaphors: the German Tradition of Intellectual History and the Complex Fabric of Language", en Javier FERNÁNDEZ SEBASTIÁN (ed.), Political Concepts and Time - New Approaches to Conceptual History, Cantabria University Press - McGraw-Hill Interamericana de Espańa, 2011, pp. 45-72.

${ }^{23}$ El término "red conceptual" es propio de la historia conceptual o Begriffsgeschichte y se debe entender desde esta.

24 Jochen BUNG, Subsumtion und Interpretation, Nomos, Baden-Baden, 2004, pp. 138-142. Véase también WIEACKER, 1983: 92.

25 Señala Atienza que es particularmente relevante su obra Europäische Literatur und lateinisches Mittelalter. Véase ATIENZA, 1997: 50.

26 "La estructura total de la jurisprudencia solamente se puede determinar desde el problema", en VIEHWEG, 1964: 129. 
Las propuestas de Viehweg se pueden considerar, sobre todo, como un rechazo del uso de la lógica formal en el Derecho ${ }^{27}$ vinculado a una revitalización de la retóri$\mathrm{ca}^{28}$. Estas propuestas y su desarrollo posterior no llegan a conformar una metodología jurídica o una teoría de la argumentación jurídica ${ }^{29}$, pero -apunta Díez Sastre- sí que tienen un valor metodológico nada despreciable por el hecho de que introducen una perspectiva cuyas explicaciones debe tener en cuenta una metodología jurídica que aspire a ser completa ${ }^{30}$. La centralidad de esta obra de Viehweg es tal, que no es inusual que en los textos las referencias a la tópica jurídica sean en realidad referencias a cómo entiende este autor la tópica jurídica ${ }^{31}$.

Viehweg presentó lo que era una tesis innovadora llamada a corregir los excesos del positivismo ${ }^{32}$ a la que se le achacó un cierto grado de indefinición en sus términos y el hecho de que estaba desconectada de la realidad de la aplicación concreta del Derecho entonces vigente ${ }^{33}$. Preguntas como, cómo opera la tópica jurídica bajo la supremacía de la ley; qué se ha de entender por tópico jurídico, o qué instancia de control supervisa las soluciones a las que llega la tópica, quedaron sin respuesta. La propuesta de Viehweg no causó indiferencia: mientras unos la acogieron positivamente, otros la rechazaron ${ }^{34}$. Entre nosotros le dedicó atención a la novedad DíezPicazo, quien publicó una traducción de Topik und Jurisprudenz a nuestro idioma con prólogo de García de Enterría ${ }^{35}$. Una década después García Amado analizó críticamente el razonamiento del alemán en su tesis doctoral ${ }^{36}$. Otros autores españoles también se ocuparían de la tópica jurídica ${ }^{37}$.

27 En este sentido, Luis RECASÉNS SICHES, "La tópica y jurisprudencia de Theodor Viehweg”, Diánoia, vol. 9, 1963, pp. 291-311 (p. 291).

${ }_{28}$ Bernard E. JACOB, "Ancient Rhetoric, Modern Legal Thought, and Politics: A Review Essay on the Translation of Viehweg's 'Topics and Law', Northwestern University Law Review, vol. 89, 4, pp. 1622-1677.

29 ATIENZA, 1997: 61.

30 DÍEZ SASTRE, 2020: 384, número marginal 52.

31 Véase a este respecto el comentario de Puy Muñoz sobre el título del estudio en español más completo de la tópica jurídica de Viehweg (Juan Antonio GARCIA AMADO, Teorias de la topica juridica, Universidad de Oviedo - Civitas, Madrid, 1988), en Francisco PUY MUŃOZ, "Una crítica de la tópica”, Anuario de Filosofía del Derecho, vol. VI, 1989, pp. 467-473 (p. 467).

32 Puede verse en la tópica jurídica una propuesta alternativa a la de los dos extremos que son el positivismo racionalista y el relativismo. Véase GARCÍA AMADO, 1988: 26.

33 Uwe DIEDRICHSEN, "Topisches und systematisches Denken in der Jurisprudenz", NJW, vol. 19, 16, 1966, pp. 697-705 (p. 702).

34 El listado de autores que han desarrollado las propuestas y el listado de sus detractores o aquellos que las han acogido con escepticismo es largo. Un largo listado de los primeros en WIEACKER, 1983: 82, nota a pie de página 5. Un largo listado de los segundos en ibid., p. 89, nota a pie de página 25.

35 VIEHWEG, 1964.

36 Fruto de su trabajo doctoral publicó: GARCÍA AMADO, 1988.

37 Destaca, por ejemplo, Puy Muńoz. En la doctrina administrativista, véase por ejemplo la mención a las tesis de Viehweg al hilo de un análisis metodológico de la doctrina constitucional alemana en Alfredo GALLEGO ANABITARTE, "Sobre el comentario constitucional: técnica y método", Revista de Estudios Politicos, vol. 121, 1962, pp. 137-168 (pp. 139, 146 y 147). 
De modo paralelo a lo que se ha indicado respecto a la tópica en el apartado inmediatamente anterior, la tópica jurídica sería un tipo específico de tópica que tendría por objeto no premisas para cualquier argumentación, sino premisas para que el jurista elabore sus argumentos. La tópica jurídica se ocuparía también del estudio del uso y naturaleza de estas premisas -los lugares comunes o tópicos jurídicos-. Al igual que ocurre con los tópicos, el tópico jurídico se expande hasta englobar realidades tan diversas como, entre otras, los principios generales, las reglas, los dictados del sentido común, las máximas, los brocardos, los refranes jurídicos, los adagios, los cánones de interpretación, los conceptos jurídicos o los estándares valorativos. Con esta noción amplia, no es desatinado plantear que la ley y el resto de normas sean un tópico más ${ }^{38}$.

\subsection{La indeterminación de la tópica}

Como se apuntaba en la introducción, y como se puede deducir de los apartados anteriores, si se examina con detenimiento el concepto mismo de tópica se capta que la tópica adolece de una indeterminación -falta de precisión- susceptible de corrección. En el núcleo de la observación se encuentra el hecho de que el mismo objeto material de la tópica-los tópicos- es muy difícil de aprehender. En tanto no se entienda con claridad qué es un tópico, no se puede entender qué es la tópica.

Ocurre así también con la tópica jurídica, razón por la cual todavía se plantea qué es un tópico jurídico y qué es la tópica jurídica ${ }^{39}$. Junto a estas dos preguntas han ido planteándose otras más que tienen que ver con su aplicación práctica y que tampoco han recibido respuesta clara. Por ejemplo: ¿cómo encaja la tópica jurídica en el sistema de fuentes?; ¿cómo se compatibiliza tópica jurídica y principio de legalidad?; ¡ cómo se compatibiliza la tópica jurídica con la idea de sistema jurídico?, o ¿cómo se resuelve un caso práctico haciendo uso de la tópica jurídica? Es difícil dar en la literatura con respuestas claras, a lo que hay que añadir que se han identificado pocos ejemplos reales en los cuales la tópica jurídica tenga relevancia práctica. Con carácter excepcional se pueden mencionar el intento de Kriele por concretar normas de Derecho constitucional no codificado ${ }^{40}$, o la sugerencia de López-Jacoiste de usar una perspectiva tópica para tratar de resolver controversias que se susciten en torno a los daños generados por una violación de derechos de la personalidad ${ }^{41}$.

Este considerable grado de indeterminación sugiere que lo primero sea analizar una vez más qué es un tópico para entender mejor qué son la tópica y la tópica jurídi-

38 Entiende García Amado que esta idea transluce en Viehweg a pesar de que este último no explicitara esta idea. Véase Juan Antonio GARCÍA AMADO, "Tópica, Derecho y método jurídico", Doxa. Cuadernos de Filosofía del Derecho, 04, 1987, pp. 161-188 (p. 174).

39 El problema de indefinición del contenido de los tópicos ya presente en los clásicos sigue sin solución clara. Así se deduce de la explicación de García Amado sobre el intento de la literatura alemana de aclarar esta indefinición. Véase GARCÍA AMADO, 1988: 126-133.

40 WIEACKER, 1983: 84.

41 José Javier LÓPEZ JACOISTE, "Una aproximación tópica a los derechos de la personalidad", Anuario de Derecho Civil, 4, 1986, pp. 1059-1120. 
ca; solo con una respuesta satisfactoria a ambas preguntas se puede intentar responder el resto. Se adelanta ya, antes de razonarlo en el apartado siguiente, que se considera aquí que la noción de tópico es irremediablemente amplia-amplísima-, y que la tópica y las funciones que esta pueda desempeñar tienen que ver con un intento por entender el intelecto humano en su proceso de alcanzar conclusiones en el terreno de lo plausible y discutible; un intento que se apoya en la metáfora del lugar común, pero que, por el hecho mismo de partir de una metáfora, debe entenderse también como una invitación a continuar investigando. La extrema apertura del término tópico - toda premisa plausible válida para la argumentación- es un rasgo inherente al concepto, y no es algo sobrevenido, como reflejaba ya su formulación aristotélica.

\subsubsection{La indeterminación de la tópica: los tópicos}

Los textos de Aristóteles no ofrecen una respuesta concluyente a la pregunta de qué es un tópico; el filósofo no se entretuvo en ello ${ }^{42}$. Se deduce que los tópicos, como premisas que son, se empleaban de herramienta en el método del razonamiento probable de la dialéctica puesto que ofrecían argumentos para el enjuiciamiento de las afirmaciones del contrincante ${ }^{43}$; pero no con carácter exclusivo, puesto que Aristóteles se refería también a otros elementos argumentales o stoicheion y empleaba ambos términos (topos y stoicheion) indistintamente ${ }^{44}$.

Su amigo y sucesor Teofrasto definió topos como un principio (arche) o elemento (stoicheion) del cual se pueden deducir principios particulares ${ }^{45}$. Siglos después, Temistio identificó topos con axioma y así definió el primero empleando la definición del segundo (apunta Ebbesen que Temistio presumiblemente identificó topos también con un cuasi-axioma $\left.{ }^{46}\right)$. Cicerón concibió un tópico como sedes argumenti, lugar en que residen los argumentos -su base o fundamento ${ }^{47}-$, y así lo hizo también Boecio ${ }^{48}$. Para este segundo el tópico se fundamenta en una proposición que es evidente de por sí - maxima propositio- y a partir de la cual se pueden deducir y contrastar proposiciones particulares que radican en diferencias específicas -locus differentia- ${ }^{49}$.

42 Lo más parecido a una definición está en su Retórica, 1403 a) 18-19: "Y llamo aquí elemento a lo mismo que lugar común, porque elemento y lugar común son nociones en donde quedan comprendidos muchos entimemas". Véase William M. A. GRIMALDI, "Studies in the Philosophy of Aristotle's Rhetoric», Hermes - Zeitschrift für Klassische Philologie”, Einzelschrift 25, 1972, pp. 115-116, y Wilhelmus Antonius DE PATER, "La fonction du lieu et de l'instrument dans les Topiques", en Gwilym Ellis Lane OWEN (ed.), Aristotle on Dialectic - The Topics - Proceedings of the Third Symposium Aristotelicum, Clarendon Press, Oxford, 1968, pp. 164-186 (p. 164).

43 GUZMÁN BRITO, 2016: 276.

44 EBBESEN, 1993: 20.

45 Ibid., p. 21.

46 Ibid., p. 23.

47 Marco Tulio CICERÓN, Tópicos, Universidad Nacional Autónoma de México, México, D. F., 2006 (traducción de Bulmaro Reyes Coria), p. 3.

48 HANSEN, 2011: 176.

49 Ibid. 
El contenido de un tópico es, de hecho, amplísimo, puesto que se refiere a todo elemento de carácter formal o material que alimenta el razonamiento de una discusión ${ }^{50}$; ya sean estos elementos inherentes o extrínsecos al propio objeto de discusión ${ }^{51}$, ya formen parte de un argumento elaborado por el dialogante o externo a él ${ }^{52}$. Cualquiera de estos puede ser un tópico. Como se ha apuntado, pueden ser un tópico, entre otros, un concepto (por ejemplo, la noción de persona), una tesis normalmente aceptada (por ejemplo que democracia y respeto a los derechos humanos están vinculados), un principio (por ejemplo, es preferible lo posible a lo imposible), una descripción (por ejemplo, el Sol es el cuerpo celeste de mayor masa del sistema solar), un esquema argumental (por ejemplo, la especie presentará las características del género al que pertenece) o una regla (por ejemplo, se sanciona a todo aquel que robe).

Complementariamente, toda discusión incurre inevitablemente en uno o varios tópicos ${ }^{53}$. Desde su origen los tópicos no se han adscrito a una única disciplina: Aristóteles los empleó como esquemas formales para una discusión en el marco de la dialéctica, y también como auxilio para la creación de silogismos retóricos ${ }^{54}$; Cicerón los usó tanto para la dialéctica como para la retórica ${ }^{55}$.

Este carácter extremadamente amplio del objeto de los tópicos se da también en los tópicos jurídicos (los lugares en los que residen los argumentos jurídicos; los elementos de una argumentación jurídica). Como se indicaba en el segundo apartado del artículo, en principio pueden ser un tópico jurídico los principios generales, las reglas, los dictados del sentido común, las máximas, los brocardos, los refranes jurídicos, los adagios, los cánones de interpretación, los conceptos jurídicos o los estándares valorativos. También una ley. Bajo esta perspectiva, son un tópico: la buena fe, el interés, el concepto de acto administrativo, la unidad de propósito del Estado, el principio de separación de poderes, el de interdicción de la arbitrariedad, el art. 1255 del Código Civil, la teoría del órgano o la regla volenti non fit inuria. En vano actuaría quien intentara redactar un catálogo exhaustivo de tópicos jurídicos ${ }^{56}$. Con razón se puede elevar otra vez la objeción de que, en realidad, casi todo es un tópico ${ }^{57}$; a lo que se le uniría la objeción de que no existen ni parámetros para diferenciar los más plausibles de los menos, ni relación de jerarquía que los ordene.

50 GRIMALDI, 1972: 119.

51 Esta distinción entre los tópicos inherentes o intrínsecos y extrínsecos al objeto de discusión es de Cicerón. Véase CICERÓN, 2006: 3.

52 Esta distinción entre argumentos propios y externos al orador es de Aristóteles.

53 CICERÓN, 2006: 25.

54 GUZMÁN BRITO, 2016.

55 Ibid.

56 Gerhard STRUCK, Topische Jurisprudenz-Argument und Gemeinplatz in der juristischen Arbeit, Athenäum, Frankfurt am Main, 1971, p. 20.

57 Como apuntaba Larenz parece que desde la tópica de Viehweg, un tópico es toda idea o punto de vista que pueden desempeñar en absoluto un papel, sea de la clase que sea, en las discusiones. Véase GARCÍA AMADO, 1988: 126. 
Procede, entonces, preguntarse si se puede definir con más precisión qué son un tópico y un tópico jurídico, o si bien se debe asumir que no se puede especificar más allá de que los tópicos son proposiciones plausibles para la argumentación de naturaleza muy diversa.

Se entiende aquí que la respuesta es más bien la segunda. Lo que el propio Aristóteles no hizo ${ }^{58}$ (explicar con precisión qué es un tópico y acotarlo a un tipo limitado de proposiciones), no se ha hecho después. Así como la explicación de Aristóteles de qué es un tópico se ha calificado de confusa ${ }^{59}$, también lo es la de autores posteriores que han sido claves para la tópica. Sirven de testimonio las sistematizaciones de los tópicos de de Cicerón ${ }^{60}$ y Boecio ${ }^{61}$. La imprecisión del tópico subsiste, como evidencia el que se haya escrito que nadie ha sido capaz de definir de modo claro y breve qué es un topos ${ }^{62}$.

En el caso de los tópicos jurídicos, la doctrina no los ha definido con precisión ${ }^{63}$. Es cierto que la diferenciación entre tópicos formales (estructuras de razonamiento de función analítica que no contienen una solución material) y materiales (aforismos, máximas o reglas ampliamente extendidas por su capacidad persuasiva que sí que contienen soluciones materiales) esclarece el hecho de que en una argumentación jurídica se puede recurrir a premisas argumentales de muy distinta índole, y que ello permite entender mejor el empleo que se hace de los tópicos en el Derecho ${ }^{64}$; pero es cierto también que a la pregunta de qué es un tópico jurídico no queda más que responder con una respuesta abierta: que son proposiciones plausibles para la argumentación jurídica. En el caso del tópico jurídico ocurre como con el resto de tópicos y su objeto se ensancha de tal modo que sume a la tópica en un grado de indeterminación alto.

\subsubsection{La indeterminación de la tópica: la tópica como intento por explicar el proceso de razonamiento en el ámbito de lo plausible y discutible}

El concepto de tópico y su extremada elasticidad están íntimamente vinculados con el propósito o misión de la tópica.

De la literatura especializada se deduce que la tópica en su origen aristotélico tenía por misión ser disciplina al servicio de la dialéctica, la cual se desenvuelve en el campo de lo probable, como complemento que se consideraba del conocimiento

58 GRIMALDI, 1972. Son particularmente importantes a este respecto las referencias de la nota a pie de página número 16. Véase también DE PATER, 1968: 164.

59 GRIMALDI, 1972:16.

60 GUZMÁN BRITO, 2016.

61 HANSEN, 2011:116.

62 Véase la referencia a Józef Maria BOCHENSKI, Formale Logik, Karl Alber, Freiburg/München, 1956 en DE PATER, 1968: 164, nota a pie de página número 2.

63 DÍEZ SASTRE, 2020: 375, número marginal 30.

64 Ibid., pp. 375-376. 
cierto (por ejemplo, como el que proporciona la metafísica) ${ }^{65}$. La tópica era propedéutica y disciplina instrumental de la scientia aristotélica ${ }^{66}$.

Es plausible, en consecuencia, entender la tópica como un intento por desentranar los mecanismos con los que nuestro entendimiento razona cuando de realidades discutibles -no evidentes de por sí- se trata. Este intento que es la tópica ha desvelado que en ese complejo proceso elaboramos nuestro razonamiento con proposiciones de muy distinta naturaleza, que gozan de inicio de cierta plausibilidad y que actúan como asideros o puntos de apoyo para nuestro razonamiento. A su vez, se puede entender la tópica, también, como una invitación a tratar de sortear las limitaciones implícitas en comparar tácitamente un complejo proceso intelectual con el tránsito físico por los puntos de un mapa cartografiado. Es decir, cabe considerar que la tópica está lastrada por el hecho de que descansa sobre una metáfora de capacidad explicativa limitada -el "lugar común"-, y que esta carencia es en sí misma una señal que indica que es necesario superar dicha metáfora.

De la primera idea del párrafo anterior se puede colegir el hecho de que no hay opción de elegir si se argumenta por medio de tópicos, sino que se hace. Para el Derecho, supone afirmar que se puede abogar por una u otra metodología jurídica, una u otra teoría de la argumentación jurídica, pero que no se puede esquivar el hecho de que en todo caso recurrimos a tópicos a la hora de pensar, de hablar, y, por tanto, de razonar jurídicamente. Desde este punto de vista, tanto el jurista que se dedica al nivel fundamental (filósofo, teórico del Derecho), el dogmático-científico ${ }^{67}$, como el que desempeña su actividad pegado al caso ${ }^{68}$ recurre involuntariamente a tópicos, sin perjuicio de que parta de una concepción positivista del Derecho, elabore sus conceptos desde la premisa de la unidad del sistema, o interprete las normas respetando una rigurosa regla de jerarquía. Contemplada de este modo, la tópica juega un papel en todas las declinaciones del actuar del jurista.

Este razonamiento aclara por qué una argumentación tópica no es incompatible con la interpretación de normas jurídicas dotadas de primacía sobre otras, con la

65 GRIMALDI, 1972:116-117. Véase también Friedrich SOLMSEN, "Dialectic without the Forms", en Gwilym Ellis Lane OWEN (ed.), Aristotle on Dialectic - The Topics - Proceedings of the Third Symposium Aristotelicum, Clarendon Press, Oxford, 1968, pp. 49-68 (pp. 52-54).

66 GRIMALDI, 1972: 116-117.

67 Una referencia expresa al hecho de que la tópica asiste a la dogmática jurídica en el momento de acordar las definiciones básicas en LERTORA MENDOZA, 1975: 211.

68 Una referencia expresa al hecho de que la tópica asiste al jurista que se ocupa de la técnica jurídica, de la decisión concreta, en Gregorio ROBLES, "La decisión en el Derecho y la tópica jurídica”, Cuadernos de la Facultad de Derecho de la Universidad de Palma de Mallorca, vol. 6, 1983, pp. 101-138 (p. 117). 
idea de sistema ${ }^{69}$, con la construcción dogmática ${ }^{70}$, con la creación de la norma de conducta $^{71}$, con la aplicación de la norma de control por medio de un razonamiento de carácter subsuntivo ${ }^{72}$, ni con la existencia de axiomas jurídicos ${ }^{73}$. Concuerda bien con lo que se ha identificado como tópica de primer grado $^{74}$, y conecta la tópica jurídica con otras tópicas. La tópica de las ciencias de la literatura ${ }^{75}$, la política, la economía, la sociología o de la teoría de la comunicación compartirían con la tópica jurídica el hecho de que todas -cada una en su campo específico-constituyen una aproximación al proceso intelectual sobre el cual se desarrolla la disciplina.

Ciertamente no corresponde aquí contrastar la idea de que pensamos y argumentamos tópicamente de modo inevitable; más bien es tarea de la filosofía del lenguaje, la teoría del conocimiento y la neurociencia ${ }^{76}$. No obstante, sí que se puede constatar que la literatura especializada que se ocupa de cómo procede nuestro pensamiento al interpretar la ley -aun a pesar de no ofrecer una respuesta clara ${ }^{77}$, , reconoce la

69 GARCÍA AMADO, 1987: 155. Más recientemente, Sobota defiende también que tópica y sistema jurídico son compatibles. Si bien se refiere a un sistema jurídico alejado de lo que la dogmática habitualmente entiende por sistema (ella se refiere a un sistema dinámico construido desde los tópicos jurídicos), se refiere a un sistema. Véase Katharina SOBOTA, "System and flexibility in law", Argumentation, vol. 5, 3, 1991, pp. 275-282 (p. 277).

${ }^{70}$ La construcción dogmática no consiste en la elaboración de un sistema cerrado, ni impide la discusión; de hecho, la dogmática se nutre de la discusión entre lo conocido y lo desconocido, lo cual equivale a admitir que cabe elaboración dogmática a partir de una discusión que recurra a tópicos. Véase Eberhard SCHMIDT-AßMANN, Verwaltungsrechtliche Dogmatik: eine Zwischenbilanz zu Entwicklung, Reform und künftigen Aufgaben, Mohr Siebeck, Tübingen, 2013, p. 4.

71 La diferencia entre la norma de conducta y la norma de control está explicada en José María RODRÍGUEZ DE SANTIAGO, Metodología del Derecho administrativo - Reglas de racionalidad para la adopción y el control de la decisión administrativa, Marcial Pons, Madrid, 2016. En este texto el autor abre la puerta al empleo de la tópica en la creación de la norma de conducta; así se entiende aquí cuando se hace referencia al empleo de normas de interpretación tópicas en este proceso. Véase ibid., p. 126.

72 Propone Hwang la tópica como puente para salvar la distancia que existe entre el texto literal de la norma y la aplicación judicial. Señala que la tópica es en este aspecto compatible con la teoría de Kelsen en tanto que ambas reconocen al juez cierta libertad a la hora de interpretar; su explicación es que la teoría pura de Kelsen apodera al juez y el pensamiento tópico le obliga a pensar desde el caso. Véase Shu-Perng HWANG, "Rechtsbindung durch Rechtsermächtigung. Ein topisches Verständnis der Reinen Rechtslehre zur Erläuterung des Verhältnisses von Richterbindung und Richterfreiheit”, Rechtstheorie, vol. 40, 1, 2009, pp. 43-70.

73 BUNG, 2004: 139.

74 La tópica de primer grado se refiere a la invención, la búsqueda de premisas, mientras que la de segundo grado se refiere a tópicos ya elaborados. Véase DÍEZ SASTRE, 2020: 374, número marginal 28.

75 Un estudio sobre los tópicos en la literatura, en Peter JEHN (ed.), Topos Forschung - Eine Dokumentation, Athenäum, Frankfurt am Main, 1972.

76 Calboli apunta la sugerencia de Chomsky de acudir a la neurología para entender la verdadera relación entre tópica y sistema. Véase Gualtiero CALBOLI, "Die Topik in Jurisprudenz und Rhetorik", en Thomas SHIRREN y Gert UEDING (eds.), Topik und Rhetorik, de Gruyter, Berlin/Boston, 2000, pp. 555-565. (p. 561). Se refiere este último a Noam CHOMSKY, Language and Thought, Moyer Bell, 1993.

77 Véase Kristina KASTENDIECK, Der Begriff der praktischen Vernunft in der juristischen Argumentation: Zugleich ein Beitrag zur Rationalisierung und ethischen Legitimation von rechtlichen Entscheidun- 
intervención de un mecanismo intelectual preexistente que proporciona los primeros lugares comunes necesarios para todo ejercicio de interpretación jurídica. Sugerencias como que nuestro lenguaje está impregnado de tópicos ${ }^{78}$ o que la tópica es meditación prelógica ${ }^{79}$ se alinean con esta apreciación. Equivalen a afirmar que el discurso jurídico opera condicionado por el hecho de que lo elaboramos a través de un razonamiento y lenguaje que se sirve siempre de lugares comunes.

Desde esta perspectiva se puede valorar la tópica jurídica como una admonición a no pasar por alto el hecho de que el método jurídico descansa sobre la base de nuestro particular modo de razonar y que este mecanismo hace acto de presencia en todos los niveles de conocimiento jurídico y se expresa siguiendo un patrón tópico y retórico. Bastaría analizar en detalle un texto jurídico para constatarlo. Por ejemplo, en una sentencia se tendrían que poder identificar tanto el carácter retórico como tópico del texto en armonía con el hecho de que el tribunal que la dicta lo haga respetando escrupulosamente el principio de legalidad. El análisis qque llevó a cabo Sobota concuerda con esta suposición. Por medio de un ejercicio que consistía en desgranar y analizar frase por frase la argumentación sobre la que un tribunal había dictado su fallo, esta autora destapó la estructura retórica que el tribunal había empleado al aplicar la ley del caso (ley del Land reguladora de los cuerpos o fuerzas del orden público) ${ }^{80}$.

En segundo lugar, la consideración de la tópica como intento de explicar el proceso de razonamiento en el ámbito de lo plausible y discutible da pie a reflexionar sobre la segunda cuestión que se planteaba en la introducción: qué funciones corresponden a la tópica en el Derecho público. Esto es objeto de la sección siguiente.

\section{LAS FUNCIONES DE LA TÓPICA JURÍDICA}

\subsection{Auxilio para entender la racionalidad de la norma positiva}

Entendida la tópica jurídica como afirmación de que el razonamiento jurídico se desarrolla necesariamente a través del uso de lugares comunes y como el análisis de estos lugares comunes, debe ser considerada como una herramienta idónea para explicar el razonamiento subyacente a las normas positivas.

\footnotetext{
gen unter Unsicherheitsbedingungen, vol. 14, Berlin-Verlag Spitz/Berlin, 2000; Hans-Joachim KOCH, "Sprachphilosophie Grundlagen der juristischen Methodenlehre", en Robert ALEXY, Hans-Joachim KOCH, Lothar KUHLEN y Helmut RÜßMANN (eds.), Elemente einer juristischen Begründungslehre, Nomos, Baden-Baden, 2003, pp. 123-133.

78 BUNG, 2004: 142.

79 GARCÍA AMADO, 1988: 175.

80 En el supuesto de estudio el Tribunal Superior Administrativo de Münster había dictado su fallo en una sentencia que tenía por objeto una reclamación en la cual el interesado había solicitado que la autoridad administrativa le dispensara refugio en invierno invocando para ello la $\$ 14$ de la OBG o ley reguladora de los cuerpos o fuerzas del orden público. Los resultados de su análisis confirman el patrón retórico de la argumentación de la sentencia. Véase Katharina SOBOTA, "Rhetorische Muster der Rechtsgewinnung am Beispiel einer Entscheidung des OVG Münster", en Topik und Rhetorik, de Gruyter, Berlin/Boston, 2000, pp. 521-540.
} 
Siguiendo la lógica de la tópica, la norma positiva debe descansar sobre una racionalidad que se nutre de lugares comunes o tópicos y, por tanto, la identificación y análisis de esos tópicos deben servir de guía para entender el sentido de esa norma positiva. La relación entre tópicos y norma positiva se podría figurar como la que existe entre falsilla y texto: los tópicos funcionan de referencia invisible al texto positivo, de patrón inadvertido de su racionalidad. La tópica sería el instrumento adecuado para descubrir los asideros del razonamiento de la norma positiva que constituyen los tópicos sobre los cuales se ha diseñado.

Por ejemplo, a través de la tópica se puede analizar la racionalidad detrás de las expresiones positivas del principio de legalidad (arts. 9.1, 53.1, 97.1, 103.1 CE) con la ayuda de tópicos jurídicos, como son algunos recogidos en la tradición anglosajona (en las obras de Bracton -nibil aliud potest rege, nisi id solum quod de iure potest - for the King can only act according to the law-, y Blackstone -rex debet esse sub lege, quia lex fecit regem - The King should be subject to the law, because the law makes the king-) ${ }^{81}$.

También por medio de la tópica se puede examinar la racionalidad detrás de los derechos de defensa del ciudadano en el procedimiento administrativo ${ }^{82}$ (derecho a ser notificado de actos que sean relevantes para su situación jurídica, el derecho a acceder a su expediente administrativo, a ser oído, a poder aportar prueba y a formular alegaciones). En este caso puede ser de interés para el análisis, por ejemplo, el tópico del que se sirvió el Dr. Bentley al impugnar una decisión del canciller de la Universidad de Cambridge que le había desprovisto de sus títulos académicos sin haberle dado la oportunidad de responder a sus acusadores, de acuerdo con el cual, hasta a Adán y Eva se les preguntó y escuchó antes de que se les desterrara del paraíso ${ }^{83}$. También serían de interés la regla de escuchar antes que decidir y otras reglas tópicas como nemine damnatur sine audiatur, nemo damnatus nisi auditus vel vocatus; audiatur et altera pars, o audi alteram partem ${ }^{84}$; todas ellas parte de lo que el Tribunal Supremo ha denominado un eterno principio de justicia ${ }^{85}$.

A la aproximación tópica de estos ejemplos se le podría oponer que contraviene el principio de legalidad en tanto que asume que el tópico jurídico precede a la ley positiva (primero tópico y después ley). A su vez, a esta objeción se le podría replicar que

${ }^{81}$ Véase Rafael DOMINGO OSLÉ (dir.), Javier ORTEGA y Beatriz RODRÍGUEZ-ANTOLÍN, Principios de Derecho Global - Aforismos jurídicos comentados, Cátedra Garrigues y Thomson Aranzadi, Cizur Menor, 2003, pp. 195-196, párrafo 576.

82 Esta denominación en Raymond ODENT, Les droits de la défense devant les jurisdictions administratives, Études et documents du Conseil d'État, Paris, 1953, pp. 50-65. La referencia está extraída de Mariano BACIGALUPO SAGGESE y José Ramón PARADA VÁZQUEZ, "Artículo 105 - Audiencia del ciudadano en procedimientos administrativos y acceso de los ciudadanos a los archivos y registros administrativos", en Óscar ALZAGA VILLAAMIL (dir.), Comentarios a la Constitución Española de 1978, Edersa, Madrid, 1998, pp. 525-545.

83 La explicación de este caso, en Allan Randolph BREWER-CARIAS, Principios del procedimiento administrativo, Civitas, Madrid, 1990, pp. 171-172.

${ }^{84}$ Las manifestaciones de este principio son numerosas. Su historia es muy rica. Véase DOMINGO OSLÉ, 2003: 50-51, párrafo 66.

85 STS de 15 de noviembre de 1934; extraída de ibid. 
supremacía o rango y racionalidad en realidad son cuestiones distintas y compatibles si se admite que existe racionalidad jurídica más allá del texto de la norma positiva. De hecho -seguiría la réplica- el mismo Derecho positivo le reconoce a los principios generales del Derecho un carácter informador del ordenamiento en el art. 1.4 del Código Civil, en la medida en que cabe interpretar esta disposición en el sentido de que es la propia ley positiva la que se abre a relacionarse con una racionalidad jurídica externa que está reflejada en los criterios o juicios de valor que una sociedad profesa en un momento histórico concreto ${ }^{86}$.

La disyuntiva entre ambas alternativas (aceptar o rechazar un análisis tópico de la ley) deja entrever que la tópica se funda y desenvuelve sobre una concepción del Derecho que admite la compatibilidad entre la norma positiva y una racionalidad jurídica extra-positiva. En el caso de que, por el contrario, se entendiera que lo jurídico y su lógica tienen su única sede en la norma positiva, la cuestión sobre la racionalidad jurídica y la supremacía se respondería en el sentido de que la ley prima y contiene en sí todo lo jurídico, también toda racionalidad jurídica; acudir a una lógica jurídica que se sitúe en tópicos no explicitados en la ley es salirse de los mismos límites de lo jurídico y no aporta elementos de análisis para un examen jurídico. Desde esta concepción del Derecho la tópica no podría desempeñar la función de auxilio interpretativo que se ha sugerido.

\subsection{Nexo entre los distintos elementos de una unidad jurídica plural}

La segunda función de la tópica está relacionada con la constatación de que los tópicos son lugares comunes, compartidos; también entre distintos ordenamientos jurídico-públicos.

Por medio de un razonamiento abstracto que los hechos corroboran, se concluye sin dificultad que entre ordenamientos jurídicos existen algunos lugares o tópicos comunes y otros que son propios y particulares de cada ordenamiento; de lo contrario los ordenamientos en cuestión no se diferenciarían los unos de los otros. Por esta razón, en el momento que se identificaran los tópicos particulares de un ordenamiento, simultáneamente, se estarían también perfilando su identidad particular dentro de un conjunto de ordenamientos.

Esta obviedad es relevante porque convierte a la identificación y análisis de tópicos jurídicos -particulares y comunes- en vía útil para perfilar los distintos elementos

86 En su comentario a este artículo Díez Picazo descarta una interpretación positivista de los principios generales del Derecho. Entiende que "no es posible ni legítimo restringir de este modo la idea de principios jurídicos" (en alusión a la interpretación positivista de los mismos). Prosigue: "Al lado de ellos [los principios generales positivos] existen también los criterios o juicios de valor que sobre la forma de resolver los conflictos de intereses y sobre la forma de organizar el ordenamiento, profesa en un determinado momento histórico una determinada sociedad". Véase Luis DÍEZ-PICAZO PONCE DE LEÓN, "Artículo 1", en Cándido PAZ-ARES RODRÍGUEZ, Luis DÍEZ-PICAZO PONCE DE LEÓN, Rodrigo BERCOVITZ y Pablo Salvador CODERCH (dirs.), Comentario del Código Civil Tomo I, Ministerio de Justicia, Madrid, 1991, p. 10, párrafo 2. 
de una unidad jurídica plural. Un ejemplo de tal unidad jurídica plural es la que representan las propuestas recientes de elaborar un ius publicum europaeum; entendiendo por este no el ordenamiento jurídico de la UE, sino el conjunto de distintos ordenamientos que conviven en el territorio de Europa (ordenamientos nacionales, de la UE, EFTA y EEA, del Consejo de Europa) ${ }^{87}$.

Si se secunda la iniciativa de elaborar un ordenamiento jurídico tal, no a partir de la voluntad de los soberanos, sino más bien sobre la base de un orden público común que convive con ordenamientos individuales de perfil propio, la elaboración a partir de tópicos es recomendable; de hecho, inevitable. La definición del estándar mínimo llamado a constituir ese orden público común ${ }^{88}$ requiere interpretar, comparar y sistematizar ${ }^{89}$, lo que necesariamente exige entender en su profundidad los tópicos de los distintos ordenamientos europeos (valores, principios y conceptos) ${ }^{90}$. Esto ocurre en toda comparación entre ordenamientos ${ }^{91}$, y ha sido clave en el método particular del cual se ha servido el TJUE para elaborar el Derecho de la UE.

Los tópicos servirían de nódulos a partir de los cuales definir el núcleo de la unidad jurídica que se trata (en este caso el ius publicum europaeum) y de entender el modo en el que cada ordenamiento particular se relaciona con los elementos de esa unidad plural. Los tópicos serían útiles para definir las particularidades de las soluciones de cada uno de los ordenamientos, lo que también llevará en ocasiones a relativizar esas soluciones nacionales puesto que en ocasiones los ordenamientos esencialmente ofrecen la misma respuesta a través de distintas fórmulas.

Esto sirve para una multitud de casos; por ejemplo, si se quisiera definir y elaborar para ese ius publicum europaeum el contenido del derecho de asilo, respecto del cual ya se ha apreciado que existen divergencias entre ordenamientos nacionales europeos, si bien menos relevantes de lo que pueda aparentar ${ }^{92}$.

${ }^{87} \mathrm{La}$ referencia es al proyecto que lidera $\mathrm{A}$. von Bogdandy desde el instituto de investigación Max Planck y que está explicado en Armin VON BOGDANDY, "The Idea of a European Public Law”, MPIL Research Paper Series, 2017-04. Esta idea de un espacio jurídico europeo más allá del Derecho de las Comunidades Europeas (después la UE) no es nueva, más bien lo contrario. Se encuentra ya en autores como von der Groeben o Mosler. Véase Hermann MOSLER, "Begriff und Gegenstand des Europarechts", Zeitschrift für ausländisches öffentliches Recht und Völkerrecht, vol. 28, 1968, pp. 481-502.

88 La identificación entre orden público y mínimo estándar en MOSLER, 1968: 497.

89 Philipp DANN, "Thoughts on a Methodology of European Constitutional Law", German Law Journal, vol. 6, 11, 2005, pp. 1453-1474 (p. 1454).

90 Así se entendía respecto al orden público de las Comunidades en Hermann MOSLER, "Der "Gemeinschaftliche Ordre Public» in europäischen Staatengruppen”, Revista Española de Derecho Internacional, vol. 21, 3, 1968, pp. 523-535 (p. 525).

91 Hans-Wolfram DAIG, "Zu Rechtsvergleichung und Methodenlehre im Europäischen Gemeinschaftsrecht", en Herbert BERNSTEIN, Ulrich DROBNIG y Hein KÖTZ (eds.), Festschrift für Konrad Zweigert, J. C. B. Mohr (Paul Siebeck), Tübingen, 1981, pp. 395-416 (pp. 406-407). Véase también Jaakko HUSA, "Überlegunten zu einer Theorie der Rechtsvergleichung als Rechtsphilosophie", Rechtstheorie, vol. 40, 2009, pp. 473-492 (pp. 479 y 486).

92 Pablo SANTOLAYA MACHETTI, El derecho de asilo en la Constitución española, Lex Nova, Valladolid, 2001, pp. 48-50. 
A través de los tópicos sobre los que descansa el derecho de asilo se podría comenzar, primero, por definir su núcleo. Una definición posible es entender que en todos los ordenamientos europeos, sobre la base de un deber de preservar la dignidad humana (el tópico de la dignidad humana), los Estados miembros han adquirido el compromiso de otorgar protección a aquellas personas que conforme a Derecho internacional cualifiquen como refugiado (el tópico del concepto internacional de refugiado), lo que supone inmunizar a las personas protegidas de la jurisdicción del Estado que persigue (el tópico de la jurisdicción de un Estado). Los tópicos de la dignidad humana, el concepto internacional de refugiado, y la jurisdicción del Estado formarían parte de ese núcleo que es parte del ordenamiento común. En segundo lugar, a partir de ese mismo núcleo se empezaría a identificar tópicos particulares en cada uno de los ordenamientos hasta llegar a completar la panorámica de tópicos o contenido común y nacional-particular. Entre las muchas particularidades se detectaría, por ejemplo, que mientras en Espańa el art. 13.4 CE encomienda a la ley la regulación de lo que se entiende es un derecho subjetivo al procedimiento de asilo y a la protección si se estima la solicitud, en Alemania, en cambio, se entiende que el ejercicio de ese derecho subjetivo está regulado en el art. 16.a) de la Ley Fundamental.

\subsection{Apoyo para la innovación y argumentación jurídicas}

Los tópicos, y por tanto la tópica, están llamados a servir de puntos desde los cuales imaginar nuevas soluciones desde lo que ya ha sido generalmente aceptado (los tópicos existentes).

Se ha manifestado que existe una relación entre tópica y memoria ${ }^{93}$, tópica y fantasía ${ }^{94}$, y también entre tópica y creatividad ${ }^{95}$; en este sentido se ha subrayado la semejanza estructural que existe entre el razonamiento tópico y el proceso de abducción de Peirce ${ }^{96}$. Los tópicos actúan de lugares de recuerdo ${ }^{97}$, de repositorio de experiencias y verdades acumuladas desde el cual la imaginación propone soluciones distintas.

93 Anselm HAVERKAMP, Renate LACHMANN y Reinhart HERZOG (eds.), Memoria - vergessen und erinnern, Fink, München, 1993. Véase también Stefan GOLDMANN, "Topik und Memoria in Sigmund Freuds Traumdeutung", Deutsche Vierteljahrsschrift für Literaturwissenschaft und Geistesgeschichte, vol. 72, 1 Supplement, 1998, pp. 157-173.

94 Esta relación entre tópica y fantasía se explica en Georg BRAUNGART, "Topik und Phantasie", en Thomas SCHIRREN y Gert UEDING (eds.), Topik und Rhetorik, Max Niemeyer, Tübingen, 2000, pp. 307-320.

95 Nicola ERNY, "Kreativität und Methode: Ein Vergleich zwischen Charles S. Peirces Begriff der Abduktion und der Methodenkonzeption von Giambattista Vico", Zeitschrift für philosophische Forschung, vol. 53, 1999, pp. 51-76.

96 Entiende este autor que el proceso de abduction o abductive inference es un tercer modo distinto al razonamiento deductivo o inductivo. Por medio de este nuestro pensamiento da con una primera hipótesis aclaratoria al investigar un problema. Se ha visto una semejanza estructural entre este proceso creativo de la primera hipótesis aclaratoria y el pensamiento tópico de Vico. Véase ibid.

97 GOLDMANN, 1998: 160. 
También en el ámbito estrictamente jurídico los tópicos son el lugar mismo de invención ${ }^{98}$. Es decir, se inventa para enfrentarse a una nueva problemática empleando los tópicos jurídicos que la memoria nos habilita, razón por la cual se ha sugerido emplear un pensamiento tópico para salvar la distancia entre el ordenamiento jurídico y los nuevos valores de una sociedad por medio de la innovación ${ }^{99}$.

Lejos de ser irrelevante, la innovación contribuye a la labor de argumentar a favor de nuevas soluciones que sean racionales y razonables. En este sentido, la oportunidad que ofrece la tópica de que se innove para alcanzar estas nuevas soluciones en el marco de un ordenamiento jurídico-positivo no hace sino reforzar la posibilidad de más argumentación en el seno de ese ordenamiento. Esto tiene un efecto positivo en el grado de implantación del Estado de Derecho, tal como ha explicado MacCormick: a mayor discusión jurídica argumentada, más Estado de Derecho ${ }^{100}$.

\subsection{Función pedagógica}

Lo anterior invita a concluir que la tópica encierra también un potencial pedagógico. El que la tópica sea útil como herramienta de análisis de la racionalidad de la norma positiva, como instrumento para la comparación entre ordenamientos, y como apoyo a la innovación argumental, trae consigo el que sea de interés como medio de estudio y aprendizaje del Derecho. El análisis a través de tópicos jurídicos debería ayudar al estudiante a familiarizarse con el contenido del Derecho, entender su racionalidad interna, comparar sus distintas manifestaciones y estimularle a seguir argumentando. No sin fundamento, la tópica estaba integrada en la tradición pedagógica dialéctico-argumentativa que unió la disputatio clásica y medieval ${ }^{101}$.

\section{CONCLUSIONES}

Una alternativa para reducir el grado de indefinición que rodea a los conceptos de tópica y tópica jurídica es indagar en el propio objeto material de la tópica: los tópicos. El ejercicio revela que las nociones de tópico y tópico jurídico (premisa plausible para la argumentación) son irremediablemente abiertas o amplias. Esto no es algo que trajera la nueva formulación de la tópica jurídica de la segunda mitad del siglo $\mathrm{xx}$, sino que ya estaba presente en la formulación aristotélica y ha subsistido hasta hoy.

Se entiende aquí que esta indeterminación del concepto de tópico está íntimamente relacionada con el hecho de que la tópica es un intento por entender y explicar cómo razona el intelecto cuando de realidades no evidentes de por sí se trata. En el

\footnotetext{
98 BRAUNGART, 2000: 307.

99 WIEACKER, 1983: 88-89.

100 Neil MACCORMICK, "Retórica y Estado de Derecho”, Isegoría, vol. 21, 1999, pp. 5-21.

101 EBBESEN, 1993: 17.
} 
desempeño de esta misión, la tópica descansa sobre la metáfora aristotélica del lugar común, lo cual revela las carencias explicativas de la metáfora, invitando así a seguir investigando en esta dirección.

Así entendida, la tópica jurídica subraya que todo razonamiento jurídico -de cualquier nivel- descansa sobre una estructura tópica y recurre inevitablemente a tópicos jurídicos. De aquí se sigue que la tópica jurídica puede desempeñar cuatro funciones en el ámbito del Derecho público: puede ser un auxilio para entender la racionalidad de la norma positiva; puede operar de nexo entre los distintos elementos de una unidad jurídica plural; puede servir de apoyo para la innovación y argumentación jurídica, y puede desarrollar una función pedagógica, tal como ya hizo en la Antigüedad clásica y Edad Media.

\section{BIBLIOGRAFÍA}

ARISTÓTELES, Ética Nicomaquea - Ética Eudemia, Gredos, Madrid, 1993 (traducción de Julio Pallí Bonet y José Ignacio García Armendáriz).

—, Tratados de lógica (Órganon) I, Gredos, Madrid, 1982 (traducción de Miguel Candel Sanmartín y José Montoya).

Manuel ATIENZA, Las razones del Derecho - Teorías de la argumentación jurídica, Centro de Estudios Constitucionales, Madrid, 1997.

Mariano BACIGALUPO SAGGESE y José Ramón PARADA VÁZQUEZ, "Artículo 105 - Audiencia del ciudadano en procedimientos administrativos y acceso de los ciudadanos a los archivos y registros administrativos", en Óscar ALZAGA VILLAMIL (dir.), Comentarios a la Constitución Española de 1978, Edersa, Madrid, 1998, pp. 525-545.

Ramón BELTRÁN CALFURRAPA, "La tópica jurídica y su vinculación argumentativa con el precedente y la jurisprudencia", Revista de Derecho de la Pontificia Universidad Católica de Valparaíso, vol. 39, 2012, pp. 587-606.

Józef Maria BOCHENSKI, Formale Logik, Karl Alber, Freiburg/München, 1956.

Armin VON BOGDANDY, "The Idea of a European Public Law”, MPIL Research Paper Series, $2017-$ 04.

Georg BRAUNGART, G., "Topik und Phantasie”, en Thomas SHIRREN y Gert UEDING (eds.), Topik und Rhetorik, de Gruyter, Berlin/Boston, 2000, pp. 307-320.

Allan Randolph BREWER-CARIAS, Principios del procedimiento administrativo, Civitas, Madrid, 1990. Jochen BUNG, Subsumtion und Interpretation, Nomos, Baden-Baden, 2004.

Gualtiero CALBOLI, "Die Topik in Jurisprudenz und Rhetorik", en SHIRREN, T. y UEDING, G. (eds.), Topik und Rhetorik, de Gruyter, Berlin/Boston, 2000, pp. 555-565.

Noam CHOMSKY, Language and Thought, Moyer Bell, 1993.

Hans-Wolfram DAIG, "Zu Rechtsvergleichung und Methodenlehre im Europäischen Gemeinschaftsrecht”, en Herbert BERNSTEIN, Ulrich DROBNIG y Hein KÖTZ (eds.), Festschrift für Konrad Zweigert, J. C. B. Mohr (Paul Siebeck), Tübingen, 1981, pp. 395-416.

Philipp DANN, "Thoughts on a Methodology of European Constitutional Law", German Law Journal, vol. $6,11,2005$, pp. 1453-1474.

Wilhelmus Antonius DE PATER, "La fonction du lieu et de l'instrument dans les Topiques", en Gwilym Ellis Lane OWEN (ed.), Aristotle on Dialectic - The Topics - Proceedings of the Third Symposium Aristotelicum, Clarendon Press, Oxford, 1968, pp. 164-186.

Uwe DIEDRICHSEN, "Topisches und systematisches Denken in der Jurisprudenz", $N J W$, vol. 19, 16, 1966, pp. 697-705. 
Luis DÍEZ-PICAZO PONCE DE LEÓN, "Artículo 1", en Cándido PAZ-ARES RODRÍGUEZ, Luis DÍEZ-PICAZO PONCE DE LEÓN, Rodrigo BERCOVITZ y Pablo Salvador CODERCH (dirs.), Comentario del Código Civil - Tomo I, Ministerio de Justicia, Madrid, 1991.

Silvia DÍEZ SASTRE, "La tópica como método en el Derecho público", Revista de Derecho Público: Teoría y Método, vol. 1, 2020, pp. 363-396.

Rafael DOMINGO OSLÉ (dir.), Javier ORTEGA y Beatriz RODRÍGUEZ-ANTOLÍN, Principios de Derecho Global-Aforismos jurídicos comentados, Cátedra Garrigues y Thomson Aranzadi, Cizur Menor, 2003.

Sten EBBESEN, "The Theory of loci in Antiquity and the Middle Ages", en Klaus JACOBI (ed.), Argumentationstheorie - Scholastische Forschungen zun den logischen und semantischen Regeln korrekten Folgerns, E. J. Brill, Leiden/New York/Köln, 1993, pp. 15-40.

Nicola ERNY, "Kreativität und Methode: Ein Vergleich zwischen Charles S. Peirces Begriff der Abduktion und der Methodenkonzeption von Giambattista Vico", Zeitschrift für philosophische Forschung, vol. 53, 1999, pp. 51-76.

Günter FRANK, "Traditionsfähigkeit antiken Wissens in der Frühen Neuzeit - Zur Überlieferung der aristotelishcen Ethica", en Thomas FRANK, Ursula KOCHER y Ulrike TARNOW (eds.), Topik und Tradition-Prozesse der Neuordnung von Wissensüberlieferungen des 13. bis 17. Jahrhunderts, V\&R unipress, Göttingen, 2007.

Manfred FUHRMANN, "Die zivelrechtlichen Beispiele in Ciceros Topik", en Thomas SCHIRREN y Gert UEDING (eds.), Topik und Rhetorik, Max Niemeyer, Tübingen, 2000, pp. 51-66.

Alfredo GALLEGO ANABITARTE, "Sobre el comentario constitucional: técnica y método", Revista de estudios politicos, vol. 121, 1962, pp. 137-168.

Juan Antonio GARCÍA AMADO, "Tópica, Derecho y método jurídico", Doxa. Cuadernos de Filosofía del Derecho, 04, 1987, pp. 161-188.

- Teorias de la topica jurídica, Universidad de Oviedo - Civitas, Madrid, 1988.

Stefan GOLDMANN, "Topik und Memoria in Sigmund Freuds Traumdeutung", Deutsche Vierteljahrsschrift für Literaturwissenschaft und Geistesgeschichte, vol. 72, 1 Supplement, 1998, pp. 157-173.

Niels Jørgen GREEN-PEDERSEN, The Tradition of the Topics in the Middle Ages - The Commentaries on Aristotle's and Boethius 'Topics', Philosophia Verlag, München/Wien, 1984.

William M. A. GRIMALDI, "Studies in the Philosophy of Aristotle's Rhetoric», Hermes - Zeitschrift für Klassische Philologie", Einzelschrift 25, 1972.

Alejandro GUZMÁN BRITO, "Dialéctica y retórica en los «topica» de Cicerón”, en Alfonso CASTRO SÁENZ y Fernando LLANO ALONSO (eds.), Cicerón - El Hombre y los Siglos, Comares, Granada, 2016, pp. 267-304.

Heine HANSEN, "Boethius' De topicis differentiis, Commentaries on", en Henrik LAGERLUND (ed.), Encyclopedia of Medieval Philosophy - Volume 1-A-L, Springer, New York, 2011, pp. 176177.

Anselm HAVERKAMP, Renate LACHMANN y Reinhart HERZOG (eds.), Memoria - vergessen und erinnern, Fink, München, 1993.

Jaakko HUSA, "Überlegunten zu einer Theorie der Rechtsvergleichung als Rechtsphilosophie", Rechtstheorie, vol. 40, 2009, pp. 473-492.

Shu-Perng HWANG, "Rechtsbindung durch Rechtsermächtigung. Ein topisches Verständnis der Reinen Rechtslehre zur Erläuterung des Verhältnisses von Richterbindung und Richterfreiheit", Rechtstheorie, vol. 40, 1, 2009, pp. 43-70.

Bernard E. JACOB, "Ancient Rhetoric, Modern Legal Thought, and Politics: A Review Essay on the Translation of Viehweg's 'Topics and Law", Northwestern University Law Review, vol. 89, 4, pp. 1622-1677.

Robert JAMISON y Joachim DYCK, Topik, Argumentation, Frommann-Holzboog, Stuttgart/Bad Cannstatt, 1983.

Peter JEHN (ed.), Topos Forschung - Eine Dokumentation, Athenäum, Frankfurt am Main, 1972.

Kristina KASTENDIECK, Der Begriff der praktischen Vernunft in der juristischen Argumentation: Zugleich ein Beitrag zur Rationalisierung und ethischen Legitimation von rechtlichen Entscheidungen unter Unsicherheitsbedingungen, vol. 14, Berlin-Verlag Spitz/Berlin, 2000. 
Hans-Joachim KOCH, "Sprachphilosophie Grundlagen der juristischen Methodenlehre", en Robert ALEXY, Hans-Joachim KOCH, Lothar KUHLEN y Helmut RÜßMANN, (eds.), Elemente einer juristischen Begründungslehre, Nomos, Baden-Baden, 2003, pp. 123-133.

Reinhart KOSELLECK, Futuro pasado, Barcelona, 1993.

Cecilia Ana LERTORA MENDOZA, "La tópica en la lógica jurídica", Anuario de Filosofía del Derecho, vol. 18, 1975, pp. 203-222.

José Javier LÓPEZ JACOISTE, “Una aproximación tópica a los derechos de la personalidad”, Anuario de Derecho Civil, 4, 1986, pp. 1059-1120.

Neil MACCORMICK, "Retórica y Estado de Derecho”, Isegoría, vol. 21, 1999, pp. 5-21.

Fiorella MAGNANO, "Cicero's Lists of Topics from Antiquity to the Early Middle Ages", Revista Espanola de Filosofía Medieval, vol. 22, 2015, pp. 85-118.

Hermann MOSLER, "Begriff und Gegenstand des Europarechts", Zeitschrift für ausländisches öffentliches Recht und Völkerrecht, vol. 28, 1968, pp. 481-502.

— "Der "Gemeinschaftliche Ordre Public» in europäischen Staatengruppen", Revista Española de Derecho Internacional, vol. 21, 3, 1968, pp. 523-535.

Raymond ODENT, Les droits de la défense devant les jurisdictions administratives, Études et documents du Conseil d'État, Paris, 1953.

Elías José PALTI, "From Ideas to Concepts to Metaphors: the German Tradition of Intellectual History and the Complex Fabric of Language", en Javier FERNÁNDEZ SEBASTIÁN (ed.), Political Concepts and Time - New Approaches to Conceptual History, Cantabria University Press - McGraw-Hill Interamericana de España, 2011, pp. 45-72.

Francisco PUY MUÑOZ, Tópica jurídica, Paredes, Santiago de Compostela, 1984.

— "Una crítica de la tópica", Anuario de Filosofía del Derecho, vol. VI, 1989, pp. 467-473.

— “Tópica jurídica y retórica jurídica: un ensayo de distinción”, Ars Iuris, vol. 31, 2004, pp. 271-303.

Luis RECASÉNS SICHES, "La tópica y jurisprudencia de Theodor Viehweg”, Diánoia, vol. 9, 1963, pp. 291-311.

Gregorio ROBLES, "La decisión en el Derecho y la tópica jurídica”, Cuadernos de la Facultad de Derecho de la Universidad de Palma de Mallorca, vol. 6, 1983, pp. 101-138.

José María RODRÍGUEZ DE SANTIAGO, Metodología del Derecho administrativo - Reglas de racionalidad para la adopción y el control de la decisión administrativa, Marcial Pons, Madrid, 2016.

Manuel Jesús RODRÍGUEZ PUERTO, "Tópica y humanismo jurídico", Anuario de Filosofía del Derecho, 2001, pp. 363-383.

Pablo SANTOLAYA MACHETTI, El derecho de asilo en la Constitución española, Lex Nova, Valladolid, 2001.

Thomas SCHIRREN y Gert UEDING (eds.), Topik und Rhetorik, Max Niemeyer, Tübingen, 2000.

Eberhard SCHMIDT-AßMANN, Verwaltungsrechtliche Dogmatik: eine Zwischenbilanz zu Entwicklung, Reform und künftigen Aufgaben, Mohr Siebeck, Tübingen, 2013.

Ferdinand SCHUPP, "Zur Geschichte der Beweistopik in der älteren griechischen Gerichtsrede", Wiener Studien, vol. 45, 1926, pp. 17-28.

Katharina SOBOTA, "System and flexibility in law", Argumentation, vol. 5, 3, 1991, pp. 275-282.

— "Rhetorische Muster der Rechtsgewinnung am Beispiel einer Entscheidung des OVG Münster", en Topik und Rhetorik, Max Niemeyer, Tübingen, 2000, pp. 521-540.

Friedrich SOLMSEN, "Dialectic without the Forms", en Gwilym Ellis Lane OWEN (ed.), Aristotle on Dialectic - The Topics - Proceedings of the Third Symposium Aristotelicum, Clarendon Press, Oxford, 1968, pp. 49-68.

Gerhard STRUCK, Topische Jurisprudenz - Argument und Gemeinplatz in der juristischen Arbeit, Athenäum, Frankfurt am Main, 1971.

Juan VALLET DE GOYTISOLO, "La jurisprudencia y su relación con la tópica en la concepción de Giambattista Vico", Revista de estudios politicos, vol. 206-207, 1976, pp. 77-136.

Theodor VIEHWEG, Topik und Jurisprudenz, C. H. Beck, München, 1953.

- Tópica y jurisprudencia, Taurus, Madrid, 1964 (traducción de L. Díez Picazo Ponce de León).

Franz WIEACKER, "Theorie des Rechtes und der Rechtsgewinnung”, en Dieter SIMON (ed.), Franz Wieacker-Ausgewählte Schriften - Band 2, Metzner, 1983, pp. 81-100. 
